\title{
Information Dependencies in MCS: Conviviality-Based Model and Metrics *
}

\author{
Patrice Caire $^{1}$ and Antonis Bikakis ${ }^{2}$ and Yves Le Traon ${ }^{1}$ \\ ${ }^{2}$ Department of Information Studies, University College London \\ a.bikakis@ucl.ac.uk \\ ${ }^{1}$ Luxembourg University, Interdisciplinary Center for Security, Reliability and Trust \\ $(\mathrm{SnT})$ \\ firstname.lastname@uni.lu
}

\begin{abstract}
Information exchange among heterogenous entities is common in most distributed systems. To facilitate information exchange, we first need ways to evaluate it. The concept of conviviality was recently introduced to model and measure cooperation among agents. In this paper, we use conviviality to model and measure information dependencies in distributed systems modeled as Multi-Context Systems. Then, we apply our findings to resolve inconsistencies among participating entities.
\end{abstract}

\section{Introduction}

Today's distributed information systems are characterized by multiple forms of cooperation between heterogeneous entities including information exchange. Examples include distributed databases, Linked Data, P2P systems, sensor networks and others. One approach to model such systems is with Multi-Context Systems $(M C S)$ [18 17/7]. MCS are logical formalizations of distributed context theories connected through bridge rules, which enable information flow between contexts.

Intuitively, MCS can be used as a representation model for any information system involving distributed, heterogeneous knowledge agents such as peer-topeer systems and distributed ontologies. Applications developed on top of MCS and other logic-based context formalizations are numerous, e.g., [22]6|23]1|2]. Such systems consist of individual entities cooperating through information sharing. Reasoning with the information they import, they derive new knowledge and take more informed decisions. These features are enabled by MCS notions of contexts, bridge rules and contextual reasoning. But, how can we evaluate the ways in which systems enable cooperation? How can we characterize a MCS based on the opportunities for information exchange that it provides to its contexts?

In previous work [11, we model conviviality in a version of MCS called Contextual Defeasible Logic. In this paper we extend our model to the general MCS model[18[17/7], and introduce measures for information dependencies based on

\footnotetext{
* The present research is supported by the National Research Fund, Luxembourg,
} CoPAInS project (code: CO11/IS/1239572). 
this notion of conviviality. Defined by Illich as "individual freedom realized in personal interdependence" 20], conviviality has been introduced as a social science concept for multiagent systems 9 to highlight soft qualitative requirements like user friendliness of systems. Conviviality is measured by counting the possible ways to cooperate, indicating degree of choice or freedom to engage in coalitions 10. The authors' coalitional theory is based on dependence networks, labeled directed graphs where nodes represent agents, and each labeled edge represents that the former agent depends on the latter to achieve some goal. In distributed information systems, individual freedom is linked to the choice of keeping personal knowledge and beliefs at the local level, while interdependence is understood as reciprocity, i.e. cooperation.

In MCS as in mutliagent systems, participating entities depend on each other to achieve their goals; in MCS, the enrichment of local knowledge. This leads to our research question: How to evaluate and improve the exchange of information in MCS with the use of conviviality model and measures? In this paper, we first propose a formal model to represent information dependencies in MCS modeled as dependence networks. Then, we define conviviality measures for MCS. Finally, we apply our findings to address inconsistency resolution in MCS.

\section{Multi-Context Systems: Definitions and Example}

We define a MCS as in [7]: A MCS $M=\left(C_{1}, \ldots, C_{n}\right)$ is a set of contexts $C_{i}=\left(L_{i}, k b_{i}, b r_{i}\right), 1 \leq i \leq n . L_{i}=\left(\mathbf{K B}_{i}, \mathbf{B} \mathbf{S}_{i}, \mathbf{A} \mathbf{C C}_{i}\right)$ is a logic, where $\mathbf{K B}_{i}$ is the set of well-formed knowledge bases of $L_{i}$ and each element of $\mathbf{K B}_{i}$ is a set of formulae; $\mathbf{B S}_{i}$ is the set of possible belief sets, and an element of a belief set is a set of formulae; and $\mathbf{A} \mathbf{C C}_{i}$ : $\mathbf{K B}_{i} \rightarrow 2^{\mathrm{BS}_{i}}$ is a function describing the semantics of the logic by assigning to each knowledge base a set of acceptable belief sets. $k b_{i} \in \mathbf{K B}_{i}$ is a knowledge base, and $b r_{i}$ a set of $L_{i}$-bridge rules over $\left(L_{1}, \ldots, L_{n}\right)$. A bridge rule can add information to a context, depending on the belief sets accepted by other contexts. An $L_{k}$-bridge rule $r$ over $L$ is of the form

$$
\begin{aligned}
r=(k: s) \leftarrow & \left(c_{1}: p_{1}\right), \ldots,\left(c_{j}: p_{j}\right), \\
& \operatorname{not}\left(c_{j+1}: p_{j+1}\right), \ldots, \boldsymbol{n o t}\left(c_{m}: p_{m}\right) .
\end{aligned}
$$

where $c_{i}, 1 \leq c_{i} \leq n$, refers to a context in $M, p_{i}$ is an element of some belief set of $L_{i}$, and $k$ refers to the context receiving information $s$. We denote by $h_{b}(r)$ the belief formula $s$ in the head of $r$. By $b r_{M}=\bigcup_{i=1}^{n} b r_{i}$ we denote the set of bridge rules in $M$. For each $H \subseteq\left\{h_{b}(r) \mid r \in b r_{i}\right\}$ it holds that $k b_{i} \cup H \in \mathbf{K B}_{L_{i}}$, i.e. bridge rule heads are compatible with knowledge bases.

Example 1. Consider a MCS M, through which three distributed software agents exchange information and classify research articles they retrieve from the web. Contexts $C_{1}-C_{3}$ in $M$ encode the knowledge of the three agents. The knowledge 
bases of the three contexts are respectively:

$$
\begin{aligned}
& k b_{1}=\{\text { sensors }, \text { corba, centralizedComputing } \leftrightarrow \neg \text { distributedComputing }\} \\
& k b_{2}=\{\text { prof } A\} \\
& k b_{3}=\{\text { ubiquitousComputing } \subseteq \text { ambientComputing }\}
\end{aligned}
$$

$C_{1}$ states in propositional logic that the article is about sensors and corba, and that centralized computing and distributed computing are opposite concepts. $C_{2}$ states (also using propositional logic) that the article is written by prof $A . C_{3}$ is an ontology about computing written in a basic description logic, according to which ubiquitous computing is a type of ambientComputing. The three agents use bridge rules $r_{1}-r_{4}$ to associate their local knowledge. For example, with $r_{1}$, $C_{1}$ classifies middleware (defined by $C_{2}$ ) as a centralized computing technology.

$$
\begin{aligned}
& r_{1}=(1: \text { centralizedComputing }) \leftarrow(2: \text { middleware }) \\
& r_{2}=(1: \text { distributedComputing }) \leftarrow(3: \text { ambientComputing }) \\
& r_{3}=(2: \text { middleware }) \leftarrow(1: \text { corba }) \\
& r_{4}=(3: \text { ubiquitousComputing }) \leftarrow(1: \text { sensors }),(2: \text { prof } B)
\end{aligned}
$$

A belief state of a MCS is the set of the belief sets of its contexts. Formally, a belief state of $M=\left(C_{1}, \ldots, C_{n}\right)$ is a sequence $S=\left(S_{1}, \ldots, S_{n}\right)$ such that $S_{i} \in \mathbf{B S}_{i}$. Intuitively, $S$ is derived from the knowledge of each context and the information conveyed through applicable bridge rules. A bridge rule of form (1) is applicable in a belief state $S$ iff for $1 \leq i \leq j: p_{i} \in S_{c_{i}}$ and for $j<l \leq m$ : $p_{l} \notin S_{c_{l}}$. Equilibrium semantics selects certain belief states of a MCS $M=\left(C_{1}, \ldots, C_{n}\right)$ as acceptable. Intuitively, an equilibrium is a belief state $S=\left(S_{1}, \ldots, S_{n}\right)$ where each context $C_{i}$ respects all bridge rules applicable in $S$ and accepts $S_{i}$. Formally, $S$ is an equilibrium of $M$, iff for $1 \leq i \leq n$,

$$
S_{i} \in \mathbf{A C C}_{i}\left(k b_{i} \cup\left\{h_{b}(r) \mid r \in b r_{i} \text { applicable in } S\right\}\right) .
$$

Example 2. In our example, $M$ has one equilibrium:

$$
S=(\{\text { sensors }, \text { corba, centralizedComputing }\},\{\text { prof } A, \text { middleware }\}, \emptyset) .
$$

\section{MCS Conviviality Property: Model and Measures}

To capture the notions of context and bridge rules, we build on [10] and define a dependence network for MCS as follows:

Definition 1 (Dependence network for MCS). A dependence network corresponding to a MCS $M$, denoted as $D N(M)$, is a tuple $\left\langle C, b r_{M}\right.$, dep, $\left.\geq\right\rangle$ where: $C$ is the set of contexts in $M ; b_{M}$ is the set of bridge rules in $M$; dep :C $\times C \rightarrow$ $2^{b r_{M}}$ is a function that is constructed as follows: for each bridge rule $r$ (in the form of (1)) in $b r_{M}$ add the following dependencies: $\operatorname{dep}\left(k, c_{i}\right)=\{r\}$ where $k$ is the context appearing in the head of $r$ and $c_{i}$ stands for each distinct context appearing in the body of $r$; and $\geq: C \rightarrow 2^{b r_{M}} \times 2^{b r_{M}}$ is for each context a total pre-order on sets of its bridge rules. 
Furthermore, based on the conviviality measures defined for multi agent systems in [10, we define the conviviality of a MCS as:

$$
\begin{aligned}
\Theta & =\sum_{L=2}^{L=|C|} P(|C|-2, L-2) \times d_{M}^{L}, \\
\Omega & =|C|(|C|-1) \times \Theta, \\
\operatorname{Conv}(M) & =\frac{\sum_{c_{i}, c_{j} \in C, i \neq j} \operatorname{coal}\left(c_{i}, c_{j}\right)}{\Omega}
\end{aligned}
$$

where $|C|$ is the number of contexts in $M, L$ is the cycle length, $P$ is the usual permutation defined in combinatorics, $\operatorname{coal}\left(c_{i}, c_{j}\right)$ for any distinct $c_{i}, c_{j} \in C$ is the number of cycles that contain the ordered pair $\left(c_{i}, c_{j}\right)$ in $D N(M)$, such that the cycles do not represent logical loops, i.e., for any participating literals, we assume no other inference ways, and $\Omega$ denotes the maximal number of pairs of contexts in cycles. $d_{M}$ is the maximum number of dependencies that a context in $M$ may have on each of the other contexts in $M$ :

$$
d_{M}=\max _{k \in M} \sum_{i=1}^{|C|} \operatorname{dep}\left(k, c_{i}\right)
$$

To summarize, the conviviality of the MCS is obtained by computing the sum of all cycles containing any ordered pair of contexts in the network, over the maximal number of contexts pairs potentially in cycles. (A value of 0 indicating an unconnected graph, i.e., no conviviality, and a value of 1 indicating a fully connected graph, i.e., maximal conviviality).

Figure 1 visualizes the dependence network corresponding to MCS $M$ in Example 1: each node corresponds to a context in $M$; dependencies are derived from the four bridge rules of $M$. Per Eq. 2 and assuming $d_{M}=1$, then $\operatorname{Conv}(M)=7 / \Omega=0.58$, where $\Omega=12$. We note that $\operatorname{Conv}(M)$ is almost maximal as adding only one bridge rule, namely from $C_{2}$ to $C_{3}$, results in a fully connected graph, i.e., maximal conviviality.

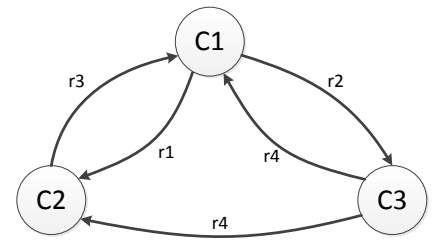

Fig. 1. The dependence network $D N(M)$ of MCS $M$ of the running example.

\section{Application: Inconsistency Resolution}

Even if contexts are locally consistent, bridge rules may render a whole MCS inconsistent. This is formally described in [7] as a lack of an equilibrium. All 
inconsistency resolution techniques proposed so far are based on the same intuition: for the entire system to be consistent again, a subset of the bridge rules causing inconsistency must be invalidated and another subset unconditionally applied. For nonmonotonic MCS, it is formally defined in 14 as diagnosis:

"Given a MCS $M$, a diagnosis of $M$ is a pair $\left(D_{1}, D_{2}\right), D_{1}, D_{2} \subseteq b r_{M}$, s.t. $M\left[b r_{M} \backslash D_{1} \cup\right.$ heads $\left.\left(D_{2}\right)\right] \not \models \perp " . D^{ \pm}(M)$ is the set of all such diagnoses, while with $M[R]$ we denote the MCS obtained from $M$ by replacing its bridge rules $b r_{M}$ with $R$; therefore $M\left[b r_{M} \backslash D_{1} \cup\right.$ heads $\left.\left(D_{2}\right)\right]$ is the MCS obtained from $M$ by removing the rules in $D_{1}$ and adding the heads of the rules in $D_{2}$.

If we deactivate the rules in $D_{1}$ and apply the rules in $D_{2}$ in unconditional form, $M$ becomes consistent. In a MCS, more than one diagnosis may restore consistency. We propose using the conviviality of the resulted system as a criterion for selecting a diagnosis. For each diagnosis we measure the conviviality of the system that is derived after applying the diagnosis, and select the diagnosis that minimally decreases conviviality. The intuition is that the system should remain as cooperative as possible. This is achieved by maximizing the number of agents involved in the derivation of a conclusion or a decision and the number of potential ways in which a conclusion may be drawn.

Diagnoses contain two types of changes applicable to bridge rules: invalidation of a rule, and unconditional application of a rule, i.e., removing the body of the rule. When invalidating or adding unconditionally rule $r$ (as defined in (1)) in a MCS $M$, all the dependencies labeled with $r$ are removed from the dependence network of $M$. Assuming that $D_{i}=\left(D_{i 1}, D_{i 2}\right)$ is a diagnosis that may be applied in $M$, and $M\left(D_{i}\right)$ is the MCS obtained $M$ after applying $D_{i}$, the optimal diagnosis is the one that maximizes the conviviality of $M\left(D_{i}\right)$ :

$$
D_{\text {opt }}=\left\{D_{i}: \operatorname{Conv}\left(M\left(D_{i}\right)\right)=\max \right\}
$$

Example 3. Consider the case, illustrated Figures 205: prof $B$ is identified by $C_{2}$ as a co-author of the paper under examination. In this case $k b_{2}$ would also contain prof $B: k b_{2}=\{$ prof $A$, prof $B\}$, which would cause an inconsistency in $k b_{1}$ due to the activation of rules $r_{4}$ and $r_{2}$. To resolve the conflict, one of the four bridge rules $r_{1}-r_{4}$ must be invalidated.

Using the diagnosis definition presented above, this is formally described as:

$$
D^{ \pm}(M)=\left\{\left(\left\{r_{1}\right\}, \emptyset\right),\left(\left\{r_{2}\right\}, \emptyset\right),\left(\left\{r_{3}\right\}, \emptyset\right),\left(\left\{r_{4}\right\}, \emptyset\right)\right\} .
$$

Figures 25 depict the four dependence networks $D N\left(M\left(D_{i}\right)\right)$, which are derived after applying $D_{i}$, where $D_{i}=\left(\left\{r_{i}\right\}, \emptyset\right)$. Dashed arrows represent the dependencies that are dropped in each $D N\left(M\left(D_{i}\right)\right)$ compared to $D N(M)$.

Following Equation 2 and the four dependence networks (Figures 205) the conviviality of each $D N$ is:

$$
\begin{aligned}
& \operatorname{Conv}\left(M\left(D_{1}\right)\right)=5 / \Omega=0.42 \text { and } \\
& \operatorname{Conv}\left(M\left(D_{j}\right)\right)=2 / \Omega=0.17 \text { with } j=2,3,4 \text { and } \Omega=12
\end{aligned}
$$

Applying $D_{1}$ (Figure 2), removes one cycle only $\left\{\left(C_{1}, C_{2}, r_{1}\right),\left(C_{2}, C_{1}, r_{3}\right)\right\}$ from the initial dependence network $D N(M)$, while applying any of diagnoses $D_{2}-D_{4}$ (Figures 355), two cycles are removed. Hence the optimal diagnosis is $D_{1}$. 


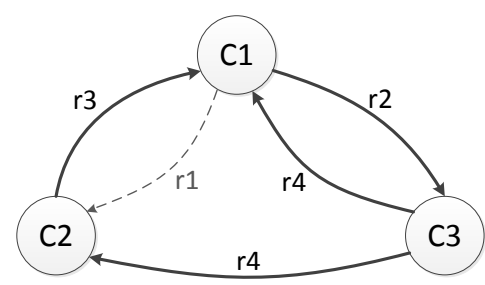

Fig. 2. $D N\left(M\left(D_{1}\right)\right)$

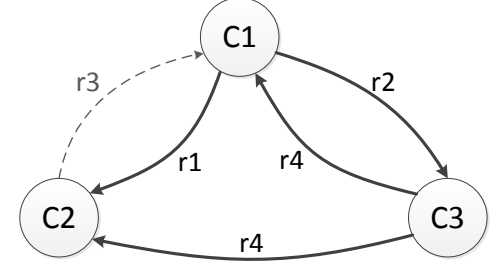

Fig. 4. $D N\left(M\left(D_{3}\right)\right)$

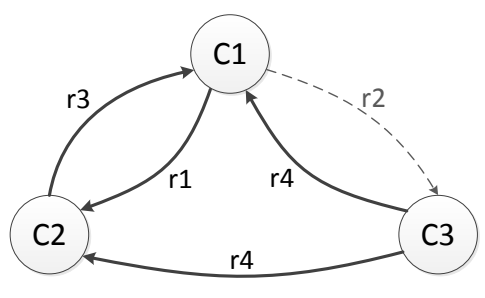

Fig. 3. $D N\left(M\left(D_{2}\right)\right)$

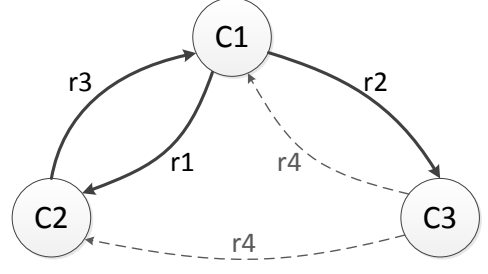

Fig. 5. $D N\left(M\left(D_{4}\right)\right)$

\section{Related Research}

The present work takes as a starting point the notion of social dependence and dependence graphs introduced by Castelfranchi et al. [13|26] and further developed, with a more abstract representation similar to ours, in Boella et al. [5]. In this context the concept of conviviality is defined as reciprocity, in Caire et al. 91012. Dependence based coalition formation is analyzed by Sichman 25], while other approaches are developed in 24 16 4. Similarly to Grossi and Turrini 19, our approach brings together coalitional theory and dependence theory within multiagent systems social cooperation study. However, our approach differs as it does not hinge on agreements, and we extend it to MCS.

Various criteria have been proposed for the choice of diagnosis in inconsistency resolution: $i$.) number of bridge rules contained in diagnosis, e.g., subsetminimal diagnoses [14; ii.) local preferences on proposed diagnoses [15]; and iii.) local preferences on contexts and provenance information in Contextual Defeasible Logic [3. Our approach differs in that we take into account a global property of the system, conviviality, with the goal of maximizing its cooperativeness. Our solution is based on the assumption of a central entity that monitors information dependences, and can be combined with any of these approaches. For example, one can apply the conviviality-based approach only to those diagnoses that comply with some local constraints representing user-defined criteria [15], or define hybrid criteria, which combine preferences on diagnoses, as explicitly defined in [15] or derived from local preferences on contexts as in [2], with conviviality-based criteria. 


\section{Conclusion and Future Work}

Multi-Context Systems (MCS) are logical formalizations of distributed context theories connected through a set of bridge rules that enable information flow between contexts. Contexts, represented as individual agents, cooperate by sharing information through their bridge rules. By reasoning on the imported information, they are then able to derive new knowledge. Hence, it is extremely useful to evaluate the ways in which system enable cooperations, and to characterize MCS based on the opportunities they provide to exchange information. In this paper, we introduce into MCS the concept of conviviality, previously proposed to model and measure potential cooperations among agents in multiagent systems. We describe how conviviality can be used to model cooperation in MCS. Based on the intuition that agents depend on the information they receive from other agents to achieve their goals (e.g. to take more informed decisions), we define dependence networks for MCS. The aim for MCS is to be as cooperative as possible, and for agents to have as many choices as possible to cooperate with other agents. We compare the conviviality of MCS, with pairwise conviviality measures. Finally we propose to use conviviality as a property of MCS to resolve inconsistencies resulting from importing mutually inconsistent knowledge from different contexts. Our approach is based on the idea that the optimal solution is the one that minimally decreases the conviviality of the system.

In further research, we plan to label dependencies among system contexts by using the heads of the rules these dependencies are derived from, rather than the rules themselves. Our intuition is that the aim of applying a rule is actually to derive the conclusion that labels the head of the rule. This will require a redefinition of dependence networks to capture both disjunction (among rules that support the same conclusion) and conjunction (among the premises of each rule). We will also address the relation between the preference order on goals, part of our dependence networks definition, and preferences on rules, contexts or diagnoses. Furthermore, we plan to combine conviviality-based inconsistency resolution with the preference-based approaches of [15] and [2], and develop hybrid criteria for inconsistency resolution, taking into account both local preferences and the conviviality of the system. Finally, we will look into how the concept and tools for conviviality can be used in other distributed knowledge models, such as Linked Data, E-connections [21] and managed MCS [8, and distributed systems (e.g. indoor intelligent environments), and study the tradeoff between conviviality and other system properties such as privacy and trust.

\section{References}

1. Antoniou, G., Papatheodorou, C., Bikakis, A.: Reasoning about Context in Ambient Intelligence Environments: A Report from the Field. In: Proceedings of KR 2010. pp. 557-559. AAAI Press (2010)

2. Bikakis, A., Antoniou, G.: Defeasible Contextual Reasoning with Arguments in Ambient Intelligence. IEEE Trans. on KDE 22(11), 1492-1506 (2010) 
3. Bikakis, A., Antoniou, G., Hassapis, P.: Strategies for contextual reasoning with conflicts in Ambient Intelligence. KIS 27(1), 45-84 (2011)

4. Boella, G., Sauro, L., van der Torre, L.: Algorithms for finding coalitions exploiting a new reciprocity condition. Logic Journal of the IGPL 17(3), 273-297 (2009)

5. Boella, G., Sauro, L., van der Torre, L.W.N.: Power and dependence relations in groups of agents. In: IAT. pp. 246-252. IEEE Computer Society (2004)

6. Bouquet, P., Giunchiglia, F., van Harmelen, F., Serafini, L., Stuckenschmidt, H.: C-OWL: Contextualizing Ontologies. In: ISWC 2003. pp. 164-179 (2003)

7. Brewka, G., Eiter, T.: Equilibria in Heterogeneous Nonmonotonic Multi-Context Systems. In: Proceedings of AAAI 2007. pp. 385-390 (2007)

8. Brewka, G., Eiter, T., Fink, M., Weinzierl, A.: Managed Multi-Context Systems. In: IJCAI. pp. 786-791 (2011)

9. Caire, P., Villata, S., Boella, G., van der Torre, L.: Conviviality masks in multiagent systems. In: AAMAS 2008, May 12-16, 2008. pp. 1265-1268 (2008)

10. Caire, P., Alcade, B., van der Torre, L., Sombattheera, C.: Conviviality measures. In: AAMAS 2011, Taipei, Taiwan, May 2-6, 2011 (2011)

11. Caire, P., Bikakis, A.: Enhancing Cooperation in Distributed Information Systems Using Conviviality and Multi-Context Systems. In: Proceedings of MIWAI 2011. LNCS, vol. 7080, pp. 14-25. Springer (2011)

12. Caire, P., van der Torre, L.: Convivial ambient technologies: Requirements, ontology and design. The Computer Journal 3 (2009)

13. Castelfranchi, C.: The micro-macro constitution of power. Protosociology 18, 208 269 (2003)

14. Eiter, T., Fink, M., Schüller, P., Weinzierl, A.: Finding Explanations of Inconsistency in Multi-Context Systems. In: Proceedings of KR 2010. AAAI Press (2010)

15. Eiter, T., Fink, M., Weinzierl, A.: Preference-Based Inconsistency Assessment in Multi-Context Systems. In: JELIA. LNCS, vol. 6341, pp. 143-155. Springer (2010)

16. Gerber, A., Klusch, M.: Forming dynamic coalitions of rational agents by use of the dcf-s scheme. In: AAMAS. pp. 994-995 (2003)

17. Ghidini, C., Giunchiglia, F.: Local Models Semantics, or contextual reasoning=locality + compatibility. Artificial Intelligence 127(2), 221-259 (2001)

18. Giunchiglia, F., Serafini, L.: Multilanguage hierarchical logics, or: how we can do without modal logics. Artificial Intelligence 65(1) (1994)

19. Grossi, D., Turrini, P.: Dependence theory via game theory. In: AAMAS 2010. pp. 1147-1154 (2010)

20. Illich, I.: Tools for Conviviality. Marion Boyars Publishers, London (August 1974)

21. Kutz, O., Lutz, C., Wolter, F., Zakharyaschev, M.: E-connections of abstract description systems. Artificial Intelligence 156(1), 1-73 (2004)

22. Lenat, D.B., Guha, R.V.: Building Large Knowledge-Based Systems; Representation and Inference in the Cyc Project. Addison-Wesley Longman Publishing Co., Inc., Boston, MA, USA (1989)

23. Sabater, J., Sierra, C., Parsons, S., Jennings, N.R.: Engineering Executable Agents using Multi-context Systems. J. of Logic and Computation 12(3), 413-442 (2002)

24. Shehory, O., Kraus, S.: Methods for task allocation via agent coalition formation. Artif. Intell. 101(1-2), 165-200 (1998)

25. Sichman, J.S.: Depint: Dependence-based coalition formation in an open multiagent scenario. J. Artificial Societies and Social Simulation 1(2) (1998)

26. Sichman, J.S., Conte, R.: Multi-agent dependence by dependence graphs. In: Procs. of The First Int. Joint Conference AAMAS 2002. pp. 483-490. ACM (2002) 